\title{
RELACIONES DE LA JUSTICIA ENTRE COMPAÑEROS Y LA JUSTICIA DEL SUPERVISOR CON EL DESEMPEÑO: UN ESTUDIO DE DIARIO
}

\section{LINKING PEER JUSTICE AND JUSTICE FROM THE SUPERVISOR TO PERFORMANCE: A DIARY STUDY}

\author{
BEATRIZ PICÓ TORIBIO ${ }^{1}$, VICENTE MARTÍNEZ-TUR ${ }^{2}$ Y \\ YOLANDA ESTREDER ${ }^{2}$
}

Cómo referenciar este artículo/How to reference this article:

Picó Toribio, B., Martínez-Tur, V. y Estreder, Y. (2019). Relaciones de la justicia entre compañeros y la justicia del supervisor con el desempeño: un estudio de diario [Linking Peer Justice and Justice from the Supervisor to Performance: A Diary Study]. Acción Psicológica, 16(1), 129-142. https://doi.org/10.5944/ap.16.1.22458

\section{Resumen}

La investigación ha estudiado el impacto de la justicia organizacional, definida como el trato que perciben los trabajadores por parte de una autoridad externa (supervisor $\mathrm{u}$ organización en su conjunto), sobre el desempeño como logro en la consecución de los objetivos del trabajo. Sin embargo, se echa en falta investigación de diario que tenga en cuenta otras fuentes de justicia más allá de la autoridad externa. Este estudio analiza la relación de dos fuentes de justicia, justicia del supervisor y justicia entre compañeros, con el desempeño de los trabajadores. Para ello, se ha realizado un estudio de diario para valorar la variabilidad en el desempeño como consecuencia del trato diario de justicia que perciben los trabajadores. Se ha realizado un

Correspondence address [Dirección para correspondencia]: Yolanda Estreder. Instituto Universitario de Investigación en Psicología de los Recursos Humanos, del Desarrollo Organizacional y de la Calidad de Vida Laboral (IDOCAL) Universitat de València, España.

Email: yolanda.estreder@uv.es

ORCID: Beatriz Picó Toribio (http://orcid.org/0000-0002-1231-6737), Vicente Martínez Tur (http://orcid.org/0000-00026388-569X) y Yolanda Estreder (http://orcid.org/0000-0003-4480-4724).

${ }^{1}$ Universitat Miguel Hernández, España.

${ }^{2}$ Instituto Universitario de Investigación en Psicología de los Recursos Humanos, del Desarrollo Organizacional y de la Calidad de Vida Laboral (IDOCAL) - Universitat de València, España.

Recibido: 24 de agosto de 2018.

Aceptado: 1 de octubre de 2018 . 
trabajo de campo con una muestra de 28 trabajadores de una empresa del sector de la automoción, durante 10 días consecutivos. Los resultados mostraron correlaciones promedio estadísticamente significativas del desempeño con la justicia del supervisor $(r=0.41, t(26)=2.29 ; p<.05)$ y la justicia entre compañeros $(r=0.44, t(26)=2.49$, $p<.05)$. Asimismo, se confirmó la existencia de una relación estadísticamente significativa y positiva entre la justicia del supervisor, percibida el primer día de medida, y el desempeño de los trabajadores medido 10 días después. En cambio, no se observó la misma relación con respecto a la justicia de los compañeros. Así, se concluye que: (a) los cambios en justicia van acompañados de cambios en el desempeño y (b) la justicia del supervisor, medida el día de comienzo, es capaz de predecir el desempeño 10 días después.

Palabras clave: Estudio de diario; Justicia entre compañeros; Justicia del supervisor; Desempeño.

\footnotetext{
Abstract

Previous research has investigated the impact of organizational justice, defined as the treatment workers receive from an external authority (supervisor or the organization as a whole), on performance as achievement of task objectives. However, there is a lack of diary studies that consider other sources of justice beyond an external authority. This study analyzes the link from two sources of justice, justice from the supervisor and peer justice, to workers' performance. To do so, we carried out a diary study to examine variability in employees' performance as a consequence of variability of daily justice treatment perceived by workers. We conducted a field survey investigation using a sample of 28 workers of a company that pertained to the automotive sector, during 10 successive days. Our findings showed significant average correlations between performance, on the one hand, and both justice from supervisor $(r=0.41, t(26)=2.29, p<.05)$ and peer justice $(r$ $=0.44, t(26)=2.49, p<.05)$. In addition, it was confirmed a significant and positive relationship between justice from the supervisor, perceived the first measurement day, and employees' performance measured 10 days after. By contrast, this relationship was not confirmed for peer jus-
}

tice. We conclude the following: a) changes in justice are associated with changes in performance; and b) justice from the supervisor, measured at the starting point, is able to predict performance ten days after.

Keywords: Diary study; Peer justice; Justice from the supervisor; Performance.

\section{Introducción}

La idea de justicia y equidad ha suscitado un gran interés para las personas de todas las culturas desde tiempos remotos. Investigadores y profesionales de diversos ámbitos, como la filosofía, la pedagogía y la sociología, entre otros, han tratado de dar una explicación a la justicia en diferentes facetas de la vida (Ryan, 1993). En el ámbito de las relaciones laborales, el tema de la justicia tiene especial relevancia, ya que estamos en una época en la que la sociedad y sus ciudadanos son muy sensibles al comportamiento responsable de las organizaciones (Pérez-Martínez y Topa, 2018). Es por ello que se ha puesto atención en cuestiones sobre los procesos de justicia en la organización y cómo esta puede afectar el comportamiento, el desempeño productivo y el compromiso laboral de los empleados (Peña-Ochoa y Durán Palacio, 2016). De hecho, la investigación ha demostrado que la justicia es un precursor muy relevante de resultados clave en las organizaciones como, entre otros, satisfacción con el trabajo, compromiso, desempeño y comportamientos de ciudadanía. Esto ocurre en el trato justo (o injusto) percibido con respecto a los supervisores y las organizaciones como autoridad externa (ver los meta-análisis de Colquitt, Conlon, Wesson, Porter y Ng, 2001; Whitman, Caleo, Carpenter, Horner y Bernerth, 2012). Los resultados aún son incipientes en el caso de la justicia entre compañeros (ver Li, Cropanzano y Molina, 2015), aunque algunos resultados apuntan a que la justicia entre compañeros podría tener un rol significativo en su desempeño (Cropanzano, Li y Benson, 2011). Sin embargo, se echa en falta estudios de diario que permitan examinar la relación de la justicia a lo largo de los días (trato que perciben cada día los trabajadores) con la variabilidad en el desempeño de los trabajadores. 
La justicia organizacional hace referencia a las percepciones que los empleados tienen sobre lo que es justo y lo que es injusto dentro la organización (De Boer, Bakker, Syroit y Schaufeli, 2002). Durante las últimas cuatro décadas, la justicia organizacional ha sido ampliamente estudiada. Inicialmente, se centró la atención en la dimensión de justicia distributiva, es decir, en cuán justa es la recompensa recibida, teniendo en cuenta la contribución que realizan los miembros de una organización (Adams, 1965). Posteriormente, varios investigadores defendieron la idea de que la justicia no podría referirse solo a lo que se obtiene del intercambio social, sino que también hace referencia a los medios por los cuales se distribuyen las recompensas o compensaciones. Esta justicia, relacionada con los métodos y procedimientos, se denomina justicia de procedimientos (Thibaut y Walker, 1975). Así, este tipo de justicia pone el acento principalmente en los determinantes estructurales. Sin embargo, la justicia de procedimientos no contempla los aspectos sociales. Esto llevó a plantear un tercer tipo de justicia, la justicia interaccional. Este tipo de justicia está asociada a la calidad del trato que perciben los trabajadores por parte de su supervisor (Bies y Moag, 1986) e incluye, a su vez, dos dimensiones: justicia interpersonal (trato digno y correcto por parte del supervisor) y justicia informacional -grado en que el supervisor comparte información con sus colaboradores- (Colquitt, 2001; Moliner; Martínez-Tur; Ramos, Peiró y Cropanzano, 2008).

El modelo de justicia organizacional de cuatro dimensiones (justicia distributiva, justicia de procedimientos, justicia interpersonal y justicia informacional) fue validado por Colquitt (2001). No obstante, cada vez son más los autores que plantean retos en la investigación sobre justicia organizacional que van más allá de la diferenciación entre facetas o dimensiones de justicia. En su influyente artículo, Ambrose y Schminke (2009) señalaron que las experiencias subjetivas de justicia en el trabajo no se correspondían con la distinción entre facetas. Más bien, los trabajadores reaccionan a partir de una percepción general de lo que es justo o no en su entorno laboral. Además, hay contextos donde determinadas facetas de la justicia no tienen mucho sentido. Por ejemplo, la justicia distributiva no tiene relevancia en la justicia que una persona percibe relacionada con el trato por parte de compañeros. Tanto el estudio de Ambrose y Schminke (2009), como otros posteriores (e.g., Nicklin, McNall, Cerasoli, Strahan y Cavanaugh, 2014), han venido a confirmar la importancia de la percepción global de justicia para los trabajadores. Otra área de investigación relevante tiene que ver con las fuentes de justicia. Tradicionalmente, la investigación se ha centrado en la percepción de justicia de los trabajadores en relación con el trato de una autoridad externa, normalmente el supervisor o la organización en su conjunto. Sin embargo, son cada vez más los autores que señalan que esto es limitado y que hay otras fuentes de justicia posibles (Lavelle, Rupp y Brockner, 2007). Una de esas fuentes son los propios compañeros de trabajo (Li y Cropanzano, 2009; Li, Cropanzano y Bagger, 2013; Li et al., 2015).

Teniendo en cuenta la temática sobre fuentes de justicia, el presente trabajo contribuye al estudio de la justicia en las organizaciones distinguiendo entre trato justo del supervisor y justicia por parte de los compañeros de trabajo. Para ello, se relacionan estas dos fuentes de justicia con una variable crítica como es el desempeño en el trabajo. Además, se pone a prueba esta relación con un diseño de estudio de diario. Esto nos permitirá valorar el grado en que la variabilidad a lo largo del tiempo en las fuentes de justicia va acompañada de variabilidad en el desempeño de los trabajadores. Este enfoque de estudio de diario se ha utilizado exitosamente en el estudio de diferentes constructos, como, por ejemplo, la relación entre familia y trabajo (Derks y Bakker, 2018). Nosotros lo ampliamos al estudio de la justicia. Esto nos permitirá tener una valoración de las percepciones de los trabajadores a lo largo de los días, ya que ellos/ellas no tienen las mismas experiencias en sus interacciones diarias con las fuentes de justicia (supervisor y compañeros).

Como se ha comentado más arriba, la investigación sobre justicia en las organizaciones pone cada vez más el acento en los agentes o fuentes que participan en la situación que se percibe como justa o injusta (Cropanzano, Byrne, Bobocel y Rupp, 2001). Se considera que es relevante para la investigación y la práctica profesional la identificación de los agentes (fuentes) que ejercen actos que se perciben como justos o injustos. Si no se considera a dichos agentes, se pueden estudiar las actitudes y comportamientos generales que la percepción de justicia genera mediante la información que aportan los distintos ti- 
pos de justicia, pero no se pueden apreciar las actitudes y comportamientos dirigidas al responsable del acto en sí (Greenbaum, Folger y Ford, 2011) ni tampoco los diferentes efectos en función de la persona o grupo que cometiera el acto de (in)justicia (Molina, 2014).

Para tener una visión más amplia y poder realizar un estudio completo de la justicia organizacional dando relevancia a la identificación del foco o fuente de justicia, Cropanzano et al. (2001) proponen la perspectiva multifoco. En el desempeño diario de los empleados se dan constantemente relaciones con diferentes entidades y personas (Blau, 1964). Estas interacciones permiten que las personas puedan desarrollar percepciones de justicia con respecto a cada una de las entidades o personas con las que entablan relaciones. Por consiguiente, la perspectiva multifoco se centra en quién es el responsable de las acciones que se perciben como justas o injustas (Molina, 2014).

La perspectiva multifoco ha empezado a dar sus frutos en la investigación. Tradicionalmente, la fuente de justicia ha sido una autoridad externa -el supervisor o la misma organización-, quien, con su comportamiento, inciden en las percepciones de justicia de los trabajadores, es decir, fuentes de autoridad con respecto a quien realiza la apreciación de justicia (Rupp, Shao, Jones y Liao, 2014). Sin embargo, la fuente de justicia puede venir de otros agentes que no tienen una autoridad externa o jerárquica sobre el individuo. Se trata de una aproximación más reciente que centra la atención en los compañeros de trabajo como fuente de justicia. Esta justicia se ha denominado justicia entre iguales - peer justice-(Lavelle et al., 2007).

Un aspecto especialmente importante desde la perspectiva multifoco es la relación entre la justicia y el desempeño. No todas las fuentes tienen por qué presentar la misma relación con el desempeño de los empleados. Los argumentos que subyacen en la relación entre justicia, tomando como fuente al supervisor o la organización, y el desempeño son muy convincentes. El trato justo de un supervisor tendrá un efecto sobre el desempeño de sus empleados, ya que, si son bien tratados, actuarán con reciprocidad y por lo tanto rendirán más. Sin embargo, no está tan claro el rol que puede tener la justicia entre iguales, ya que la investigación de esta fuente es aún muy incipiente. No obstante, cuando los trabajadores perciben que hay un trato justo entre ellos, es probable que transfieran esta percepción a una mayor disposición positiva para mostrar un desempeño adecuado. La justicia entre compañeros se refiere al clima existente entre los miembros del equipo de trabajo y no a la percepción que un individuo en particular tiene en relación a cómo es tratado por sus compañeros ( $\mathrm{Li}$ et al., 2015). Cuando los trabajadores perciben que entre ellos se tratan con justicia, entonces es posible un esfuerzo mayor en términos de desempeño (Cropanzano et al., 2011).

Así pues, una primera contribución del presente estudio consiste en examinar hasta qué punto la justicia entre iguales "compite" con la justicia más tradicional (trato por parte del supervisor) en la predicción del desempeño. Trataremos de esclarecer, pues, cuál de las dos (o si ambas) tiene relaciones estadísticamente significativas con el desempeño de los trabajadores. Una segunda contribución del presente estudio es el uso de un diseño de investigación de "estudio de diario". La gran mayoría de estudios sobre justicia se han llevado a cabo con estudios transversales (un solo momento de medida) o con pocos momentos de medida. En cambio, la investigación internacional reconoce cada vez más la importancia de realizar estudios de diario. Por una parte, estos estudios nos permiten valorar la variabilidad en el desempeño como consecuencia del trato diario de justicia que perciben los trabajadores. Por otra parte, nos permite poner a prueba un test más sólido de los dos tipos de justicia, ya que podemos examinar hasta qué punto un trato justo es capaz de predecir el desempeño un número de días después. La consideración de un estudio de diario nos obliga a centrar la atención en la justicia interpersonal. Esto es debido a que el trato respetuoso por parte del supervisor y de los compañeros puede variar a lo largo de los días. Por el contrario, los otros tipos de justicia (salarios, procedimientos, etc.) son más estables y no sería esperable tener variabilidad a lo largo de los días.

Teniendo en cuenta todo lo anterior, el presente trabajo tiene dos objetivos. En primer lugar, se examina hasta qué punto la variabilidad a lo largo de los días en la percepción de justicia, tanto por parte del supervisor como entre los compañeros de trabajo, lleva aparejada una variabilidad en el desempeño de los trabajadores, entendido como consecución de los objetivos de la tarea. Es de esperar una co- 
rrelación positiva a lo largo de los días. Es decir, los días en que se percibe un trato justo debería observarse también un mejor desempeño. En segundo lugar, se pone a prueba hasta qué punto la justicia percibida el primer día de medida, tanto por parte del supervisor como entre compañeros, es capaz de predecir el desempeño que perciben los trabajadores el último día de medida. Esta estrategia nos permite evitar los problemas relacionados con la varianza compartida por uso de método común y conseguir resultados más sólidos (Podsakoff, McKenzie, Lee y Podsakoff, 2003). En otras palabras, al poner en relación dos medidas lo más distantes entre sí evitamos las relaciones artificialmente altas que se pueden producir cuando el participante contesta a las dos variables en el mismo momento temporal.

\section{Método}

\section{Participantes}

Para la realización de este estudio se ha contado con una muestra de conveniencia de 28 trabajadores pertenecientes al sector de la automoción. De los 28 sujetos, 13 eran mujeres y 15 hombres, con edades comprendidas entre los 21 y 61 años, con una edad media de 44 años. Todos los sujetos pertenecían al departamento de producción. Realizaban tareas similares de producción tales como ejecución de los procesos establecidos de acuerdo con los estándares de la empresa, provisión de información sobre problemas encontrados y otras cuestiones del trabajo, cumplimiento de los niveles establecidos de coste y calidad y participación en reuniones vinculadas a la producción. Contestaron a un cuestionario durante 10 días laborables consecutivos. Se obtuvieron, por tanto, 280 medidas $(28 \times 10)$. En línea con la investigación internacional con estudio de diario (e.g., Hulsheger, Lang y Schewe, 2015), ha primado el número de medidas (días x participantes) sobre el tamaño general de la muestra de participantes.

\section{Material}

La herramienta de evaluación que se ha utilizado ha sido un cuestionario elaborado para la ocasión, que pre- tende medir la relación entre la justicia por parte del supervisor inmediato, la justicia por parte de los compañeros de trabajo y el desempeño. El cuestionario se respondió de forma anónima. Para poder estudiar la evolución de las respuestas, respetando el anonimato, cada trabajador eligió un color y un número favorito, los cuales debían ser los mismos todos los días que respondían al cuestionario. También había que señalar la fecha en la que se realizaba, para luego poder ordenarlos cronológicamente, así como la edad y el sexo. En total, se recogieron las medidas durante 10 días laborales consecutivos para cada participante.

Se ha buscado el diseño de un cuestionario corto que fuera viable para la contestación repetida durante 10 días por parte de los participantes. De hecho, la investigación internacional recomienda el uso de medidas abreviadas, e incluso medidas mono-ítem, en diseños de estudio de diario (Ohly, Sonnentag, Niessen y Zapf, 2010). La justicia por parte de supervisores y la justicia por parte de los compañeros se midió, en cada caso y para este estudio, con un ítem típico de justicia interpersonal (Colquitt, 2001) que se refería al trato respetuoso. En el caso de la justicia percibida con respecto a los supervisores, el ítem fue: "Hoy, mi supervisor/a me ha tratado con respeto", mientras que en la medida de justicia entre compañeros se utilizó el siguiente ítem: "Hoy, el trato entre nosotros (compañeros) ha sido respetuoso". La escala de desempeño contaba con tres ítems: "Hoy he cumplido con los objetivos de mi trabajo"; "Hoy he cumplido con todas las exigencias de mi trabajo"; y "Hoy he desempeñado bien mi trabajo" (Goodman y Svyantek, 1999). El coeficiente de fiabilidad Alpha de Cronbach para la medida de desempeño osciló entre 0.70 y 0.93 a lo largo de los días, siendo satisfactorio en cada uno de los 10 días de medida. La escala de respuesta de todos los ítems es Likert de siete puntos, y variaba de 1-"Totalmente en desacuerdo" a 7-"Totalmente de acuerdo".

\section{Procedimiento}

Al final de la jornada laboral, se administraba el cuestionario a cada uno de los 28 empleados que participaba en el estudio, para que estos los cumplimentaran de forma voluntaria y anónima. Este procedimiento se siguió a lo 


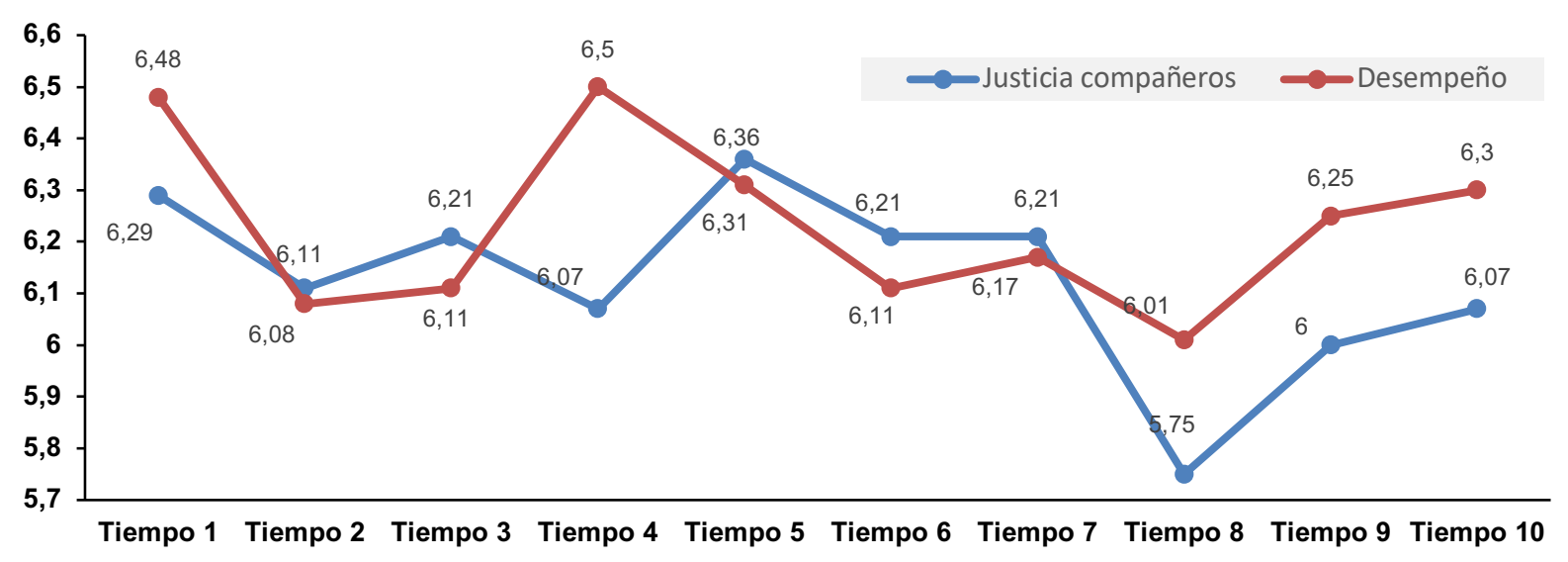

Figura 1. Relación entre la Justicia entre compañeros y el desempeño a lo largo de 10 días.

largo de diez días, como se ha indicado anteriormente. Cada cuestionario se corresponde con cada uno de los días de trabajo que tenían que realizar el mismo, siempre al finalizar la jornada laboral, y depositarlo en el buzón de la oficina de Recursos Humanos.

\section{Análisis de datos}

Para observar la relación entre las tres variables, se llevó a cabo la realización de los cálculos descriptivos de éstas, calculando la media (promedio aritmético), la desviación típica (promedio de fluctuación de los datos respecto a la media) y las correlaciones de Pearson de las va- riables justicia entre compañeros, justicia con el supervisor y desempeño. Por último, se realizaron análisis de regresión para examinar hasta qué punto la justicia percibida el primer día era capaz de predecir el desempeño el último día. Todos los análisis se realizaron a nivel individual.

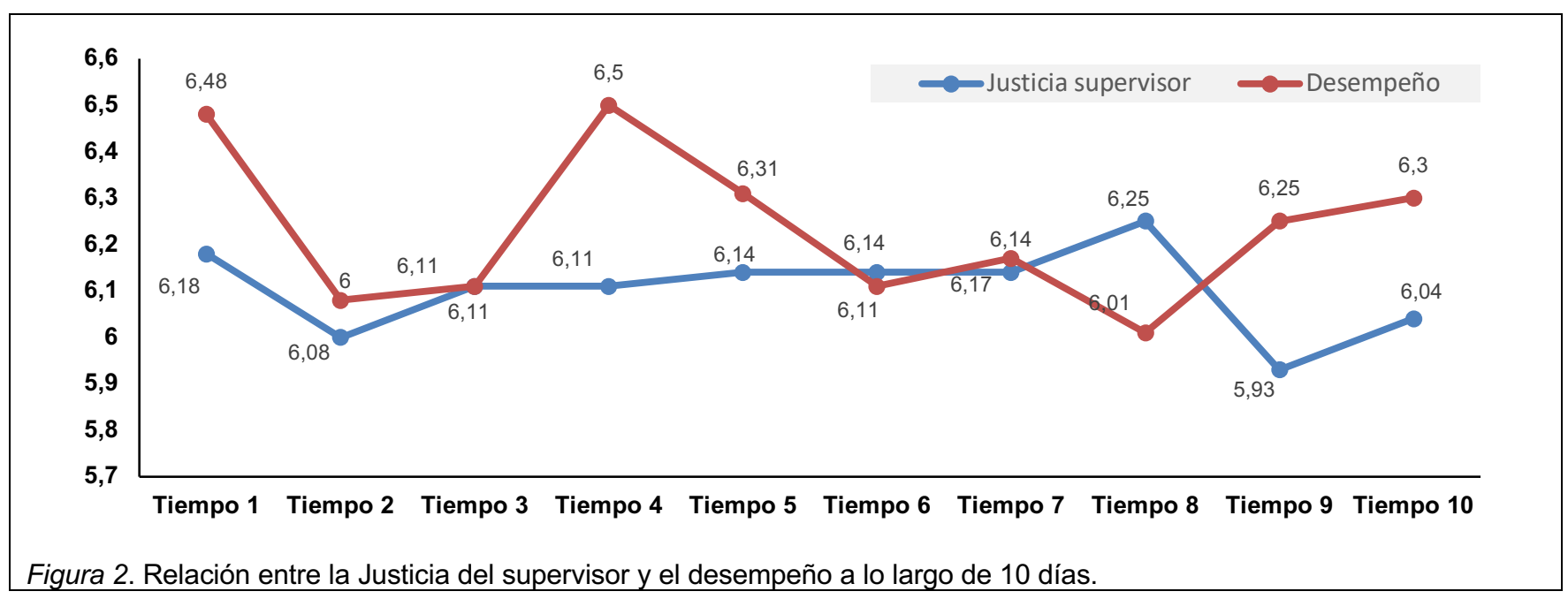


Por una parte, se confirma que hay variabilidad a lo largo de los diez días tanto en justicia como en desempeño $\mathrm{y}$, por otra parte, podemos observar, a nivel descriptivo, que cambios en justicia van acompañados de cambios en desempeño. No obstante, existen otras variables que deben estar influyendo (e.g., condiciones físicas de un día en particular) ya que hay momentos donde los cambios en desempeño no se corresponden con cambios en justicia (e.g., el cuarto día es muy evidente) ya que el desempeño se incrementa y, sin embargo, la justicia, sobre todo entre compañeros, desciende. Los coeficientes de correlación nos permiten tener un análisis más preciso (ver Figura 3 y Figura 4).
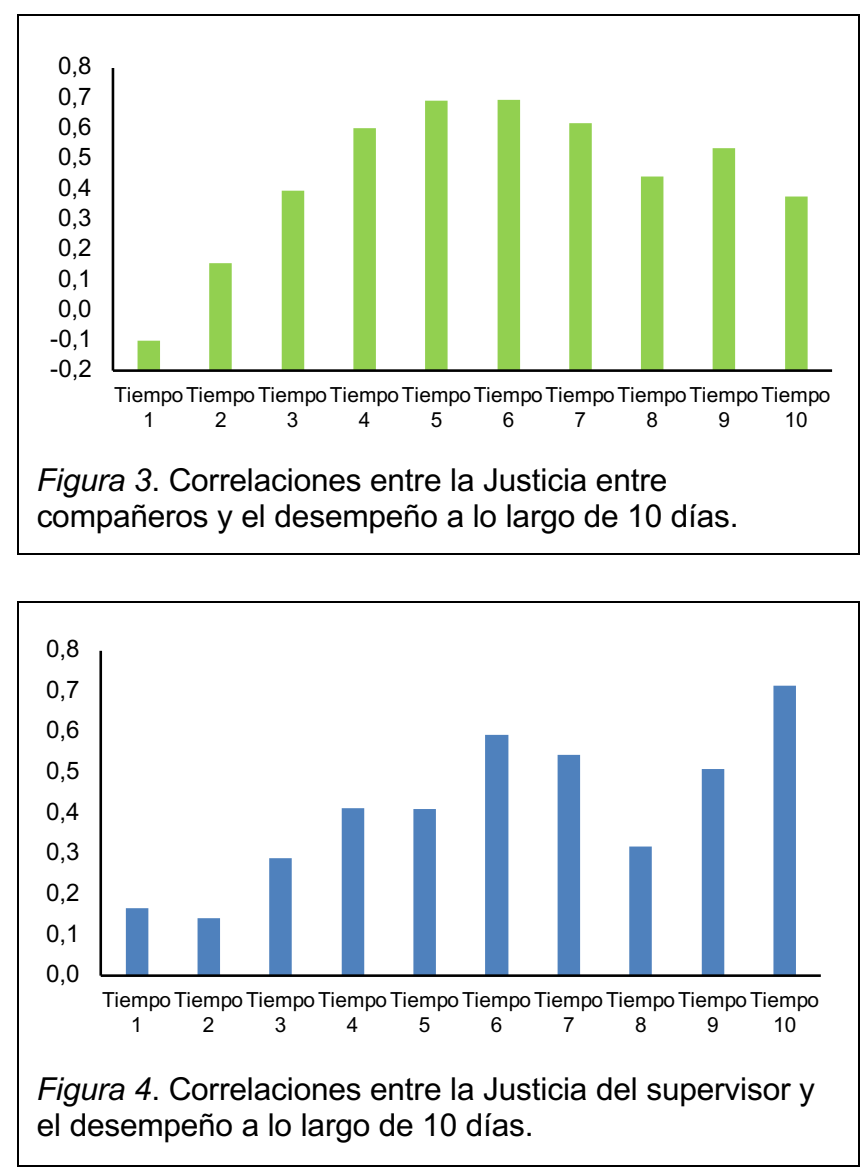

La correlación promedio de Pearson a lo largo de los días es de 0.41 para justicia del supervisor (rango 0.14 a $0.72), \quad \mathrm{y}$ resultó estadísticamente significativa $(t(26)=2.29, p<.05)$, mientras que para justicia de com- pañeros es de 0.44 (rango de -0.1 a 0.69), y también resultó estadísticamente significativa $(t(26)=2.49, p<.05)$. En general se confirma que un trato justo por parte del supervisor y un trato justo por parte de compañeros explican la variabilidad en desempeño a lo largo de los días. Es decir, el trato justo un día en cuestión lleva aparejado un incremento del desempeño ese día en la gran mayoría de los días.

Se presentan los resultados de los análisis de regresión en la Tabla 1 y en la Tabla 2. En la Tabla 1, podemos observar que la relación entre la justicia entre compañeros y el desempeño no es estadísticamente significativa en el tiempo.

Tabla 1

Resultados del análisis de regresión justicia entre compañeros y desempeño

\begin{tabular}{cccc}
\hline & $\boldsymbol{\beta}$ & & $\mathrm{EE}$ \\
\hline Paso 1 & & & \\
Edad & 0.01 & & 0.02 \\
$\begin{array}{c}\text { Sexo } \\
\mathrm{R}^{2}\end{array}$ & 0.10 & 0.01 & 0.36 \\
$\begin{array}{l}\text { Paso 2 } \\
\text { Justicia compañeros } \\
\quad \mathrm{R}^{2}\end{array}$ & 0.09 & & \\
\hline
\end{tabular}

Nota. EE = Error Estándar.

En cambio, en la Tabla 2 se puede observar que la justicia del supervisor el primer día sí muestra una relación estadísticamente significativa con el desempeño el último día evaluado. Está relación se observa una vez controladas las variables sexo y edad de los participantes.

Tabla 2

Resultados del análisis de regresión justicia del supervisor y desempeño

\begin{tabular}{cccc}
\hline & $\beta$ & & EE \\
\hline Paso 1 & & & \\
Edad & 0.01 & & 0.02 \\
Sexo & 0.10 & & 0.36 \\
$\mathrm{R}^{2}$ & & 0.01 & \\
$\begin{array}{l}\text { Paso 2 } \\
\text { Justicia supervisor } \\
\quad \Delta \mathrm{R}^{2}\end{array}$ & $0.25^{*}$ & & 0.13 \\
\hline
\end{tabular}

Nota. EE = Error Estándar. 


\section{Discusión}

Los resultados obtenidos en este estudio confirman la relación de la justicia con el desempeño, ya que cambios en la justicia acompañan a cambios en el desempeño. Esto se ha visto a nivel descriptivo, pero también calculando las correlaciones cada día. Es de destacar que las correlaciones promedio entre justicia y desempeño, a lo largo de los días, eran superiores a 0.40 y estadísticamente significativas: $r=0.41, t(26)=2.29, p<.05$ para justicia de supervisores y $r=0.44, t(26)=2.49 . p<.05$ para justicia entre compañeros). Así, se ha observado que la variabilidad en el desempeño a lo largo de los días estaba relacionada con cómo variaba la percepción de justicia entre los trabajadores. Esto confirma la estrecha relación que la investigación y el desempeño de los trabajadores (Colquitt et al., 2001; Whitman et al., 2012). Sin embargo, se observa que la capacidad para predecir el desempeño al final del periodo estudiado (relación entre justicia medida el primer día y desempeño medido el último día) no es igual para los dos tipos de justicia: entre compañeros y del supervisor. En la investigación previa ha predominado el estudio de la justicia por parte de una figura de autoridad externa -supervisor u organización en su conjunto- (ver Cropanzano et al., 2001). No obstante, son cada vez más los autores que proponen incorporar la justicia entre compañeros para explicar el comportamiento de los trabajadores (e.g., Li et al., 2015). De este modo, hemos incorporado esta fuente de justicia para observar hasta qué punto es capaz de predecir el desempeño. Nuestros resultados indican que existe una relación estadísticamente significativa en el tiempo (desde T1 a T10) entre la justicia del supervisor y el desempeño de los empleados, mientras que, por el contrario, la relación no es significativa entre la justicia entre compañeros y el desempeño. Esto indica que el trato justo de un supervisor tiene mayor peso en el desempeño de los empleados que el trato justo entre compañeros, ya que, si los empleados son bien tratados por su supervisor, actuarán con reciprocidad a más largo plazo. En cierta medida, los resultados muestran una lógica clara. El desempeño es un resultado esperado por parte de la organización y de sus responsables. El empleado se siente obligado a mostrar un mejor desempeño al verse tratado de manera justa por el supervisor (figura de autoridad que representa a la organización) pero esto no ocurre cuando se trata de la justicia emitida por los compañeros. La norma de reciprocidad
(Gouldner, 1960) funciona para el trato justo que se percibe en la relación con el supervisor (Moliner, MartínezTur, Peiró, Ramos y Cropanzano, 2013), pero no se confirma la idea de que un trato justo entre compañeros se traslada a una disposición más positiva hacia el desempeño por parte de los trabajadores (Li et al., 2015; Molina et al., 2015).

Los resultados también indican que hay variabilidad a lo largo de los diez días tanto en justicia como en desempeño. Esto era de esperar, ya que nuestra atención estaba centrada en la justicia interpersonal, por lo que era bastante probable que el trato respetuoso por parte del supervisor y los compañeros pudiese variar a lo largo de los días. Por el contrario, los otros tipos de justicia (salarios, procedimientos, etc.) son más estables y no sería esperable tener variabilidad a lo largo de los días. El uso de un diseño de investigación de "estudio de diario", cada vez más recomendado en la investigación en psicología (Ohly et al., 2010), nos permite poner a prueba un test más sólido de los dos tipos de justicia y por lo tanto poder observar cómo un trato justo por parte del supervisor es capaz de predecir el desempeño de los empleados un número de días después, mientras que, por el contrario, la justicia entre compañeros no es capaz de predecir el desempeño unos días más tarde. La consideración de diferentes momentos de medida posee una ventaja muy relevante en la investigación en psicología. En muchas ocasiones, la misma persona contesta a todas las cuestiones de estudio en un único momento de medida, lo cual tiende a producir un incremento artificial de la magnitud de la relación entre variables (Podsakoff et al., 2003). Una de las maneras de corregir esto consiste en ampliar el tiempo que transcurre entre las medidas de las variables consideradas, en nuestro caso justicia y desempeño.

Si tenemos en cuenta los resultados de este estudio, podemos concluir que el trato justo por parte de los supervisores en las organizaciones es un factor relevante. El desempeño de los empleados será mayor si estos perciben que son tratados justamente por su supervisor. Por tanto, es necesario que las empresas presten gran interés en que sus empleados sean tratados con respeto, cortesía y dignidad, por parte de sus supervisores. De este modo, trato justo y desempeño del empleado son compatibles. 
En cuanto a la justicia entre compañeros, como ya hemos comentado, los resultados muestran que tiene cierta relación, en términos descriptivos y con el cálculo de las correlaciones, con el desempeño, aunque en menor medida que la justicia del supervisor cuando se trata de predecir el desempeño 10 días después. Esto no quiere decir que no debamos prestarle atención. En futuros estudios, se puede examinar hasta qué punto el papel de la justicia entre compañeros puede ser más complejo y tener un efecto indirecto a medio plazo a través, por ejemplo, de la creación de un clima positivo en los equipos de trabajo.

Por último, señalar que este trabajo posee ciertas limitaciones que convendría mejorar en futuras investigaciones. En primer lugar, nuestro estudio de diario se ha centrado en la justicia interpersonal. Sería interesante conocer la influencia de los cuatro tipos de justicia, sobre el desempeño de los empleados, aunque esto supusiera otro diseño temporal que permitiera observar variabilidad a lo largo del tiempo en otros tipos de justicia más estables. Asimismo, dada la falta de relación entre justicia entre compañeros y desempeño, sería recomendable explorar hasta qué punto hay otros procesos mediadores que pudieran tener un papel relevante. Otra de las limitaciones es que nuestra muestra pertenece a una única empresa, por lo que sería ampliar el abanico de empresas y sectores en futuros estudios. También es una limitación del estudio el reducido tamaño muestral, debido al número de trabajadores del departamento que participó en el estudio. En este sentido, nuestra investigación es un esfuerzo inicial sobre la relación entre justicia y desempeño con estudio de diario. El tamaño muestral no permite análisis transnivel (Hox, 2010) por lo que sería muy conveniente la ampliación de la muestra en futuros estudios, permitiendo análisis más complejos. Finalmente, nuestro estudio se ha centrado en la percepción del desempeño como consecución de los objetivos de las tareas. En futuros estudios, sería conveniente contar con otras medidas que permitieran tener una visión más rica y completa del desempeño, que fuese más allá de una apreciación subjetiva. Así, además de la percepción del desempeño por parte del propio trabajador sería interesante contar también con indicadores objetivos de desempeño.

A pesar de estas limitaciones, este estudio representa un esfuerzo inicial por usar los estudios de diario en la in- vestigación de las fuentes de justicia en las organizaciones y su relación con el desempeño de los trabajadores. Los resultados parecen mostrar que conseguir un contexto laboral justo en el día a día estimula el desempeño de los trabajadores, y que la justicia del supervisor logra predecir dicho desempeño varios días después. Así pues, conseguir un lugar de trabajo justo no sólo es positivo en sí mismo, sino que, además, es compatible con el desempeño de los trabajadores.

\section{Referencias}

Adams, J. S. (1965). Inequity in Social Exchange. En L. Berkowitz (Ed.), Advances in Experimental Social Psychology (vol. 2, pp. 267-299). New York: Academic Press.

Ambrose, M. L. y Schminke, M. (2009). The Role of Overall Justice Judgments in Organizational Research: A Test of Mediation. Journal of Applied Psychology, 94(2), 491-500. https://doi.org/10.1037/a0013203

Bies, R. J. y Moag, J. F. (1986). Interactional Justice: Communication Criteria of Fairness. En R. J. Lewicki, B. H. Sheppard y M. H. Bazerman (Eds.), Research on Negotiation in Organizations (pp. 4355). Greenwich, CT: JAI Press.

Blau, P. M. (1964). Exchange and Power in Social Life. New York, NY: John Wiley.

Colquitt, J. A. (2001). On the Dimensionality of Organizational Justice: A Construct Validation of a Measure. Journal of Applied Psychology, 86, 386400. https://doi.org/10.1037/0021-9010.86.3.386

Colquitt, J. A., Conlon, D. E., Wesson, M. J., Porter, C. O. L. H. y Ng, K. Y. (2001). Justice at the Millennium: A Meta-Analytic Review of 25 Years of Organizational Justice Research. Journal of Applied Psychology, 86(3), 425-445. https://doi.org/10.1037//0021-9010.86.3.425 
Cropanzano, R., Byrne, Z. S., Bobocel, D. R. y Rupp, D. E. (2001). Moral Virtues, Fairness Heuristics, Social Entities, and other Denizens of Organizational Justice. Journal of Vocational Behavior, 58, 164-209. https://doi.org/10.1006/jvbe.2001.1791

Cropanzano, R., Li, A. y Benson, L. (2011). Peer Justice and Teamwork Process. Group \& Organization Management, 36(5), 567-596. https://doi.org/10.1177/1059601111414561

Cropanzano, R., Rupp, D. E., Mohler, C. J. y Schminke, M. (2001). Three Roads to Organizational Justice. En G. R. Ferris (Ed.), Research in Personnel and Human Resources Management (vol. 20, pp. 1113). New York: JAI Press.

De Boer, E., Bakker, A., Syroit, J. y Schaufeli, W. (2002). Unfairness at Work as a Predictor of Absenteeism. Journal of Organizational Behavior, 23, 181-197. https://doi.org/10.1002/job.135

Derks, D. D. y Bakker, A. B. (2018). Daily Spillover from Family to Work: A Test of the Work-Home Resources Model. Journal of Occupational Health Psychology, 23(2), 237-247. https://doi.org/.1037/ocp0000073

Goodman, S. A. y Svyantek, D. J. (1999). Person Organization Fit and Contextual Performance: Do Shared Values Matter. Journal of Vocational Behavior, 55(2), 254-275. https://doi.org/10.1006/jvbe

Greenbaum, R. L., Folger, R. y Ford, R. C. (2011). Moral Contracts. En S. W. Gilliland, D. D. Steiner y D. P. Skarlicki (eds.), Emerging Perspectives on Organizational Justice and Ethics (pp. 199-221). Charlotte, NC: Information Age Publishing.

Gouldner, A. W. (1960). The Norm of Reciprocity: A Preliminary Statement. American Sociological Review, 25(2), 161-164. https://doi.org/0.2307/2092623
Hox, J. (2010). Multilevel Analysis: Techniques and Applications ( $2^{\mathrm{a}}$ ed.). New York, NY: Routledge.

Hulsheger, U. R., Lang, J. W. B. y Schewe, A. F. (2015). When Regulating Emotions at Work Pays Off: A Diary and an Intervention Study on Emotion Regulation and Customer Tips in Service Jobs. Journal of Applied Psychology, 100(2), 263-277. https://doi.org/10.1037/a0038229

Lavelle, J. J., Rupp, D. E. y Brockner, J. (2007). Taking a Multifoci Approach to the Study of Justice, Social Exchange, and Citizenship Behavior: The Target Similarity Model. Journal of Management, 33(6), 841-866. https://doi.org/10.1177/0149206307307635

Li, A. y Cropanzano, R. (2009). Fairness at the Group Level: Justice Climate and Intraunit Justice Climate. Journal of Management, 35(3), 564-599. https://doi.org/10.1177/0149206308330557

Li, A., Cropanzano, R. y Bagger, J. (2013). Justice Climate and Peer Justice Climate: A Closer Look. Small Group Research, 44(5), 563-592. https://doi.org/10.1177/1046496413498119

Li, A., Cropanzano, R. y Molina, A. (2015). Fairness at the Unit Level: Justice Climate, Justice Climate Strength, and Peer Justice. En Russell, S. Cropanzano y Maureen L. Ambrose (eds.), The Oxford Handbook of Justice in the Workplace (pp. 137-164). New York, NY: Oxford University Press.

Molina, A. (2014). Justicia organizacional en el sector servicios: El impacto de "peer justice" sobre el desempeño de la organización, la calidad percibida por los clientes y el bienestar de los empleados [Organisational Justice in the Service Sector: The Impact of Peer Justice on the performance of the organisation, the quality perceived by clients and the well-being of employees] (Tesis doctoral). Universitat de València, Valencia. 
Moliner, C., Martínez-Tur, V., Ramos, J., Peiró, J. M. y Cropanzano, R. (2008). Organizational Justice and Extrarole Customer Service: The Mediating Role of Well-Being at Work. European Journal of Work and Organizational Psychology, 17(3), 327-348.

Molina, A., Moliner, C., Martínez-Tur, V., Cropanzano, R. y Peiró, J. M. (2015). Unit-level Fairness and Quality within the Health Care Industry: A JusticeQuality Model. European Journal of Work and Organizational Psychology, 24(4), 627-644, https://doi,org/10,1080/1359432X,2014,960401

Moliner, C., Martínez-Tur, V., Peiró, J. M., Ramos, J. y Cropanzano, R. (2013). Perceived Reciprocity and Well-Being at Work in Non-Professional Employees: Fairness or Self-Interest? Stress \& Health, 29(1), 31-39. https://doi.org/10.1002/smi.2421

Nicklin, J. M., McNall, L. A., Cerasoli, C. P., Strahan, S. R. y Cavanaugh, J. A. (2014). The Role of Overall Organizational Justice Perceptions within the Four-dimensional Framework. Soc Just Res, 27, 243-270. https://doi.org/10.1007/s11211-014$0208-4$

Ohly, S., Sonnentag, S., Niessen, C. y Zapf, D. (2010). Diary Studies in Organizational Research. An Introduction and some Practical Recommendations. Journal of Personnel Psychology, 9, 79-93. https://doi.org/10.1027/1866-5888/a000009

Peña-Ochoa, M. y Durán Palacio, N. M. (2016). Justicia organizacional, desempeño laboral y discapacidad [Organizational Justice, Work Performance, and Disability]. Revista Colombiana de Ciencias Sociales, 7(1), 201-222.

Pérez-Martínez, S. A. y Topa, G. (2018). Percepciones sobre la responsabilidad social corporativa de las empresas: Relación con las actitudes y conductas de sus clientes [Perceptions of Corporate Social
Responsibility: Relationship with Customers' Attitudes and Behaviors]. Acción Psicológica, 15(1), 103-120. https://doi.org/10.5944/ap.15.1.22003

Podsakoff, P. M.,McKenzie, S. B., Lee, J.-Y. y Podsakoff, N. P. (2003). Common Method Bias in Behavioral Research: A Critical Review of the Literature and Recommended Remedies. Journal of Applied Psychology, 88(5), 879-903. https://doi.org/10.1037/0021-9101.88.5.879

Rupp, D. E., Shao, R., Jones, K. S. y Liao, H. (2014). The Utility of a Multifoci Approach to the Study of Organizational Justice: A Meta-Analytic Investigation into the Consideration of Normative Rules, Moral Accountability, Bandwidth-Fidelity, and Social Exchange. Organizational Behavior and Human Decision Processes, 123(2), 159-185. https://doi.org/10.1016/j.obhdp.2013.10.011

Ryan, A. (1993). Justice. Oxford, England: Oxford University Press.

Thibaut, J. W. y Walker, L. (1975). Procedural justice: A psychological Analysis. Hillsdale, NJ: Lawrence Erlbaum Associates.

Whitman, D. S., Caleo, S., Carpenter, N. C., Horner, M. T. y Bernerth, J. B. (2012). Fairness at the Collective Level: A Meta-Analytic Examination of the Consequences and Boundary Conditions of Organizational Justice Climate. Journal of Applied Psychology, $\quad 97(4), \quad$ 776-791. http://dx.doi.org/10.1037/a0028021 


\title{
LINKING PEER JUSTICE AND JUSTICE FROM THE SUPERVISOR TO PERFORMANCE: A DIARY STUDY
}

\author{
BEATRIZ PICÓ TORIBIO ${ }^{1}$, VICENTE MARTÍNEZ-TUR ${ }^{2}$, AND \\ YOLANDA ESTREDER ${ }^{2}$
}

\section{EXTENDED SUMMARY}

\section{Introduction}

Organizational justice is a key element into labor relationships due to the society and its citizens are very sensitive to the responsible behavior of organizations (PérezMartínez \& Topa, 2018). Researchers have analyzed questions about the processes of justice in the organization and how can affect behavior, performance and organizational commitment of employees (Peña-Ochoa \& Durán Palacio, 2016). In fact, previous findings have shown that justice is a very important predictor of main organizational results such as, among others, job satisfaction, organizational commitment, performance and citizenship behaviors. This occurs in the fair (or unfair) treatment perceived with respect to supervisors and organizations as an external authority (see metanalysis of Colquitt et al., 2001; Whitman et al., 2012). The results are still incipient in the case of peer justice (see Li et al., 2015), although some results suggest that peer justice could have a significant role in their performance (Cropanzano et al., 2011). However, there is a lack of daily studies that allow us to examine the relationship of justice over the days (treatment that workers receive each day), with the variability in the performance of workers.

Organizational justice refers to the employees' perceptions about what is fair and what is unfair within the organization (De Boer et al., 2002). During the last four decades, organizational justice has been widely studied. The model of four-dimensional organizational justice (distributive justice, procedural justice, interpersonal justice and informational justice) was validated by Colquitt (2001). However, more and more authors are raising challenges in research on organizational justice that go beyond the differentiation between facets or dimensions of justice. In their influential article, Ambrose and Schminke (2009) pointed out that the subjective experiences of justice at work did not correspond to the distinction between facets. Rather, workers react from a general perception of what is fair or not in their work environment. In addition, there are contexts where certain facets of justice do not make much sense. Both the study by Ambrose and Schminke (2009), as well as later ones (e.g., Nicklin et al., 2014), have confirmed the importance of the global perception of justice for workers. Another relevant area of research is about the sources of justice. Traditionally, research has focused on the perception of workers' fairness in relation to the treatment of an external authority, usually the supervisor or the organization as a whole. However, more and more authors point out that this is limited and that there are other possible sources of justice (Lavelle et al., 2007). One of those sources is the co-workers themselves (Li \& Cropanzano, 2009; Li et al., 2013; Li et al., 2015).

Based on the abovementioned rational, the present study contributes to the study of justice in organizations, distinguishing between fair treatment of the supervisor and fairness on the part of coworkers (peer justice). To do this, these two sources of justice are related to a critical variable such as job performance. In addition, this relationship is tested with a diary study design. This will allow us to assess the degree to which variability over time in sources of justice is accompanied by variability in the performance of workers. This approach to diary study has been successfully used in the study of different constructs, 
such as, for example, the relationship between family and work (Derks \& Bakker, 2018). We extend it to the study of justice. This will allow us to have an assessment of the perceptions of the workers throughout the days, since they do not have the same experiences in their daily interactions with the sources of justice (supervisors and peers).

\section{Method}

\section{Sample}

To carry out this study, we have had a convenience sample of 28 workers (13 were women and 15 men) of a company that pertained to the automotive sector. The range of age varied between 21 and 61 years, with a mean of 44 years. All subjects belonged to the production department and they carried out similar production tasks. They answered a questionnaire for 10 consecutive working days (it was answered anonymously at the end of the working day). Therefore, 280 measurements ( $28 \times 10)$ were obtained. In line with the international research with diary study (e.g., Hulsheger et al., 2015), the number of measures (days x participants) over the overall size of the sample of participants has prevailed.

\section{Material}

The evaluation tool that has been used has been an adhoc questionnaire, which aims to measure the relationship between justice by the immediate supervisor, peer justice and performance. The questionnaire was answered anonymously. In order to study the evolution of the answers, respecting the anonymity, each worker chose a favorite color and number, which should be the same every day that they answered the questionnaire. It was also necessary to indicate the date in which it was carried out, to be able to sort them chronologically, as well as age and sex. In total, the measures were collected for 10 consecutive workdays for each participant.

We have looked for the design of a short questionnaire that was viable for the contestation repeated for 10 days by the participants. In fact, international research recommends the use of abbreviated measures, and even single-item measures, in diary study designs (Ohly et al., 2010). Justice by supervisors and peer justice were measured, in each case and for this study, with a typical item of interpersonal justice (Colquitt, 2001) that referred to respectful treatment. In the case of perceived justice with respect to the supervisors, the item was: "Today, my supervisor has treated me with respect", while in the measure of peer justice the following item was used: "Today, the deal between us (partners) has been respectful". The performance scale had three items: "Today I have fulfilled the objectives of my work"; "Today I have fulfilled all the demands of my work"; and "Today I have done my job well" (Goodman \& Svyantek, 1999). The Cronbach Alpha reliability coefficient for the performance measure ranged between 0.70 and 0.93 over the days, being acceptable in each of the 10 days of measurement. The response scale of all the items is Likert of seven points, and varied from 1- "Totally disagree" to 7- "Totally agree".

\section{Procedure}

At the end of the working day, the questionnaire was administered to each of the 28 employees who participated in the study, so that they could complete them voluntarily and anonymously. This procedure was followed for ten days, as indicated above. Each questionnaire corresponds to each of the workdays that they had to do, always at the end of the workday, and deposit it in the mailbox of the Human Resources office.

\section{Data analysis}

To observe the relationship between the three variables, we carried out the descriptive analysis of these, calculating the mean (arithmetic average), the standard deviation (average fluctuation of the data with respect to the mean) and the Pearson correlations of the variables peer justice, justice with the supervisor and performance. Finally, regression analyzes were conducted to examine the extent to which the perceived justice on the first day was able to predict performance on the last day. All analyzes were performed at the individual level. 


\section{Results}

Our findings showed significant average correlations between performance, on the one hand, and both justice from supervisor $(r=0.41, t(26)=2.29, p<.05)$ and peer justice $(r=0.44, t(26)=2.49, p<.05)$. In addition, it was confirmed a significant and positive relationship between justice from the supervisor, perceived the first measurement day, and employees' performance measured 10 days after. By contrast, this relationship was not confirmed for peer justice.

\section{Discussion}

Taking into account the results of this study, we can conclude that fair treatment by supervisors in organizations is a relevant factor. The performance of employees will be greater if they perceive that their supervisor treats them fairly. Therefore, it is necessary for companies to take great interest in having their employees treated with respect, courtesy and dignity by their supervisors. In this way, fair treatment and employee performance are compatible. Regarding peer justice, as we have already mentioned, the results show that it has a certain relationship, in descriptive terms and with the calculation of correlations, with performance, although to a lesser extent with justice of the supervisor when it comes to predicting the performance 10 days later. This does not mean that we should not pay attention to it. In future studies, it can be examined to what extent the role of peer justice can be more complex and have an indirect effect in the medium term through, for example, the creation of a positive climate in work teams.

Finally, note that this study has certain limitations that should be improved in future research. First, our diary study has focused on interpersonal justice. It would be interesting to know the influence of the four types of justice, on the performance of employees, even if this meant another temporary design that allowed observing variability over time in other more stable types of justice. In addition, given the lack of relationship between peer justice and performance, it would be advisable to explore to what extent there are other mediating processes that could have a relevant role. Another limitation is that our sample belongs to a single company, so it would be to expand the range of companies and sectors in future studies. The small sample size is also a limitation of the study, due to the number of workers in the department that participated in the study. In this sense, our research is an initial effort on the relationship between justice and performance with daily study. The sample size does not allow cross-level analysis (Hox, 2010) so it would be very convenient to extend the sample in future studies, allowing more complex analyzes. Finally, our study has focused on the perception of performance as achievement of the objectives of the tasks. In future studies, it would be advisable to have other measures that would allow a richer and more complete vision of the performance that went beyond a subjective appreciation. Thus, in addition to the perception of performance by the worker himself, it would be interesting also to have objective performance indicators.

Despite the study limitations, this study represents an initial effort to use daily studies in the investigation of the sources of justice in organizations and their relation to the performance of workers. The results seem to show that achieving a fair work context in the day to day stimulates the performance of workers, and that the supervisor's justice manages to predict such performance several days later. Thus, getting a fair workplace is not only positive in itself, but also compatible with the performance of workers. 